\title{
Sequencing Size Selection Range
}

National Cancer Institute

\section{Source}

National Cancer Institute. Sequencing Size Selection Range. NCI Thesaurus. Code

C153365.

The minimum and maximum lengths of the DNA fragments selected for inclusion in a sequencing library. 